\title{
Nitric Oxide and Respiratory Helminthic Diseases
}

\author{
Antonio Muro ${ }^{1}$ and José-Luís Pérez-Arellano ${ }^{2}$ \\ ${ }^{1}$ Laboratorio de Inmunología Parasitaria y Molecular, Centro de Investigación de Enfermedades Tropicales de la Universidad de \\ Salamanca (CIETUS), Campus Miguel de Unamuno s/n, 37007 Salamanca, Spain \\ ${ }^{2}$ Unidad de Enfermedades Infecciosas y Medicina Tropical, Servicio de Medicina Interna, Hospital Universitario de Las Palmas de \\ Gran Canaria, Facultad de Medicina, Universidad de las Palmas de Gran Canaria, 35080 Las Palmas de Gran Canaria, Spain
}

Correspondence should be addressed to Antonio Muro, ama@usal.es

Received 3 July 2009; Accepted 12 October 2009

Academic Editor: Luis I. Terrazas

Copyright ( 2010 A. Muro and J.-L. Pérez-Arellano. This is an open access article distributed under the Creative Commons Attribution License, which permits unrestricted use, distribution, and reproduction in any medium, provided the original work is properly cited.

\begin{abstract}
Nitric oxide (NO) is a very simple molecule that displays very important functions both in helminths (mainly those involved in respiratory pathology) and in mammalian hosts. In this paper we review four issues related to interaction of NO and lung helminthic diseases. Firstly, we evaluated data available on the NO synthesis and release by helminths and their biological role. Next, we summarized the effect of antigens obtained from different phases of the biological cycle on NO production by host mammalian cells (mainly from human sources). Thirdly, we revised the evaluation of NO on the biological activities and/or the viability of respiratory helminths. Lastly, the deleterious consequences of increased production of NO during helminthic human infection are detailed.
\end{abstract}

\section{Introduction}

Many helminths are frequently in contact with pulmonary cells inducing lung injury (Box 1) which has different clinical manifestations (Table 1). During the helminthic lung infection there are many molecular interactions between inflammatory cells and helminths. Among them, nitric oxide $(\mathrm{NO})$ is an important molecule involved in the pathogenesis of these diseases (Box 2).

The interaction between $\mathrm{NO}$ and helminths could be dissected in different issues that are reviewed in this paper: firstly, the NO production by different stages of the biological cycle of these parasites; secondly, the effect of helminth antigens on NO release by host cells; thirdly, the effect of NO on some helminths; and lastly, the pathological consequences derived from the increased NO production in helminthic diseases.

\section{Do Helminths Produce NO?}

There is fragmentary information available about the NO production by different helminths. This is due to the different techniques utilized in these studies (e.g., immunohisto- chemistry, histochemical techniques (NADPH diaphorase), functional studies (use of inhibitors)) together with the absence of systematic studies in all helminth species.

NO-Synthase (NOS) enzymes or NO-activity-derived products (nitrites or nitrotyrosine) have been detected in different locations of adult worms. This is the case of neural NOS (nNOS) which it has been mainly found in the nervous tissue of Schistosoma mansoni [1] and, in the same way, inducible NOS (iNOS) in the parenchyma of Schistosoma mansoni and in the subtegument of Schistosoma japonicum [2]. Additionally, NO release in living schistosomes has been demonstrated by Kohn et al. [3], suggesting a functional role for the NO in the schistosome biology.

The presence of NOS has been also demonstrated in other helminths; for example, its functional activity has been detected in different nervous structures (central, peripheral, and enteric) and the hypodermis of the nematode Ascaris suum [4-6]. Similar distribution appears in Toxocara canis [7]. NOS is located in the muscular wall from adult worms in Brugia malayi, Dirofilaria immitis and Acanthocheilonema vitae filariae $[8,9]$. Expression of endothelial NOS (eNOS) has been detected in the cuticle and stichocytes from Trichinella britovi [10]. Nitrites have been detected in the 
Respiratory system is formed by two components: Lungs (principally responsible for the respiratory function) and chest wall, which allows adequate ventilation. The lungs are constituted by a system of conduction (bronchia and bronchioles) and a system of gaseous exchange (alveoli), assembled by connective tissue and surrounded by the visceral pleura. The alveoli are the structures responsible for the gaseous exchange, located in the wall of the respiratory bronchioles or in the peripheral region of the alveolar sacs. Alveoli are not isolate structures but are found physically joined among themselves, to the bronchioles and to the visceral pleura and connected to other pulmonary structures by Köhn's pores and Lambert's canals which communicate the alveolar sacs to the terminal bronchioles. Structurally two cell types form the alveolar region, type I and type II neumocytes. Although the numerical proportion between both is $1: 2$, the type I neumocytes cover an area 25 times greater than that of type II neumocytes. Participation in the gaseous exchange is the principal function of the type I neumocytes, whereas the type II neumocytes are responsible for the production of surfactant and cellular regeneration after aggression to the alveolar region. The alveolar macrophages are the principal defensive element of the alveolointerstitial region. In their surface are expressed receptors related to adhesion (CD11a/CD18, CD29/CD49, CD54), to the capture of antigens joined to immunoglobulins, complement, or proteins (CD14, CD16, CD32, CD64, Cd11b/CD18, $\mathrm{CD} 11 \mathrm{c} / \mathrm{CD} 18)$, to the capture of nutrients (CD71), and related to the response to cytokines (CD25, CD115-130) and molecules related to the antigenic presentation (HLAII). Alveolar macrophages could be activated by different stimuli (e.g., $\gamma \mathrm{IFN}$ ) and their response involved release of lysosomal enzymes, production of free oxygen radical, and generation of nitric oxide.

Box 1

TABLE 1: Clinical-biological pattern in pulmonary helminthic diseases.

\begin{tabular}{|c|c|c|c|}
\hline Clinical-biological patterns & Subtypes & Syndrome & Parasites \\
\hline \multirow{6}{*}{ Pulmonary mass or nodule } & & & E. granulosus \\
\hline & & & D. immitis \\
\hline & & & Ascaris sp. \\
\hline & & Löffler’s Syndrome & A. duodenale \\
\hline & No extrapulmonary & & N. americanus \\
\hline & 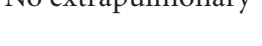 & & W. bancrofti \\
\hline \multirow[t]{5}{*}{ Pulmonary infiltrates } & & Tropical eosinophilia & B. malayi \\
\hline & & & B. timori \\
\hline & & Katayama's Syndrome & Schistosoma sp. \\
\hline & Extrapulmonary & Visceral larva migrans & Toxocara sp. \\
\hline & & Hyperinfection Syndrome & Strongyloides sp. \\
\hline Miliar pattern/Pulmonary hypertension & & & Schistosoma sp. \\
\hline Pleural effusion & & & Paragonimus sp. \\
\hline Inflammatory myopathy & & & Trichinella sp. \\
\hline
\end{tabular}

hydatid liquid of fertile Echinococcus granulosus, although NOS expression has not been identified yet [11]. Finally, expression of iNOS and nNOS has been detected in the parenchyma and nervous structures of the filariform larvae from Strongyloides venezuelenesis (unpublished data). Moreover, NOS expression has also been demonstrated in other phases, such as eggs, sporocysts, and cercariae of Schistosoma sp. [2] and other structures as oocytes, spermatozoids, and embryonic forms of Brugia malayi [8].

$\mathrm{NO}$ is essential in different biological functions of some helmints; among them neurotransmission at the nervous and muscular levels is the best characterized $[1,4,12]$. $\mathrm{NO}$ is involved in muscular relaxation and is a mediator of a variety of neuropeptides on their activity in the ionic channels (e.g., of potassium) [13]. The detection of
NOS in embryonic stages of different helminths and the role of this mediator in other species (e.g., Drosophila) suggest that NO plays an important role in the control of proliferation and differentiation during embryogenesis [8]. In this sense, helminths seem as other invertebrates (i.e., Drosophila spp, I obsolete) in the involvement of $\mathrm{NO}$ as an essential development signal [14-16]. Finally, NO plays an important role in the detoxification of free radicals of oxygen at least in two types of helminths: firstly, the hemoglobine from Ascaris lumbricoides functions as a deoxygenase, using NO to eliminate oxygen $[17,18]$; secondly, NO stimulates the haemooxygenase activity in the nurse cell of Trichinella britovi, as an useful strategy to control the "oxidative burst" that takes place after the invasion of the muscular cells [10]. 
Nitric oxide $(\mathrm{NO})$ is a very simple molecule that performs different biological functions, both in the intraand extracellular space. This molecule is generated from the amino acid L-arginine, by the action of the nitric oxide synthase (NOS), enzymes which, in the presence of oxygen produces L-citrulin and nitric oxide. These enzymes require three substrates for their action: arginin, NADPH, and oxygen and five cofactors: haem group, tetrahydrobiopterin, calmodulin, FMN (flavin mononucleotide), and FAD (flavin adenine dinucleotide). The study of the metabolism of NO could be performed using inhibitors of the NOS. The main inhibitors used in the practice are analogues of arginine, such as L-NAME (N-nitroarginin methyl ester) and L-canavanin with an irreversible effect and L-NMMA ( $\mathrm{N}^{\mathrm{w}}$-monomethyl-Larginine) with a reversible action. Four types of NOS are described: neuronal NOS (nNOS), endothelial NOS (eNOS), mitochondrial NOS (mNOS), and inducible NOS (iNOS). Constitutive enzymes (nNOS and eNOS) requires for activation a calcium dependent union to calmodulin. In the case of iNOS, the union between the enzyme and calmodulin is not calcium dependent. The synthesis of iNOS is stimulated by bacterial substances, for example, lipopolysaccharide (LPS) and by different cytokines released by macrophages or Th1 lymphocytes. The main physiological actions of NO are (i) the control of vascular tone (arterial vasodilation and inhibition of adhesion and aggregation of platelets), (ii) neurotransmission (learning and memory at the central nervous system and relaxation of visceral smooth muscle in the peripheral nervous system), and (iii) pathogenesis and control of infectious and parasitic diseases. NO can be measured directly or indirectly. The direct type of measurement is difficult to perform, since it is a molecule with a very short half life and rapidly diffuses to the tissues to perform its action. The indirect methods more employed for the NO detection are Griess technique and NOS expression. Nitrites and/or nitrates can be detected as products of its metabolism by Griess technique. NOS detection can be performed by immunocytochemistry or Western Blot and gene expression by RT-PCR or real-time PCR.

Box 2

\section{Do Helminths Induce NO Production by the Host Cells, and if So, How They Do It?}

There are several pieces of evidence indicating that helminths induce NO production by the host cells. Firstly, the presence of products derived from this molecule (mainly nitrites and nitrates) has been directly measured in the sera of humans affected by different parasitic infections, such as hydatidosis and schistosomiasis where it has been shown an increase in the production of nitrites $[19,20]$. Specifically, a positive correlation was found between $\mathrm{NO}$ production and severe clinical data in hydatidosis [21].

A second group of studies was constituted by the detection or different NO related products in experimental models of parasite infection. Thus, mice infected with $E$. multilocularis demonstrated an increase in iNOS expression in peritoneal macrophages [22]. Furthermore, in a hepatic schistosomiasis model induced by eggs of $S$. japonicum, an increase on the expression of iNOS has been detected in inflammatory cells (e.g., neutrophils, macrophages, Kuppfer cells), and hepatocytes [23]. Also, an increase in the concentration of nitrites in serum, accumulation of nitrosilated products (nitrotyrosine) and expression of iNOS in inflammatory hepatic cells has been detected in experimental toxocariosis [7]. Expression of transmural iNOS in jejunum has been observed in experimental infection with Trichinella spiralis larvae [24] and expression of iNOS has been also observed in cells of inflammatory infiltrates around larvae in skeletal muscles of Trichinella spiralis-infected mice [25]. In vivo administration of $S$. mansoni antigens (e.g., p38) plus IL-2 gives rise to granulomas in which an increase in the expression of iNOS is detected [26]. Our research group studied the concentration of nitrites in the urine of mice experimentally infected with Strongyloides venezuelensis. Mice infected with $S$. venezuelensis had high values of nitrites at the second postinfection day, corresponding with the passage of the larvae through the lungs (unpublished data).

Thirdly, the stimulation of NO production induced by parasite antigens has been evaluated by cell cultures. The results obtained by our research group demonstrated three well-differentiated aspects, according to the helminth species, the type of antigen from the same parasite, and the kind of inflammatory cell utilized in the experiment (Figure 1). Firstly, there are opposed effects on the NO production according to the groups of helminths studied. We compared the effect of Echinococcus granulosus- and Echinococcus multilocularis-defined metacestode structural and metabolic antigens on the NO production by rat alveolar macrophages. Our results showed that none of these antigens could stimulate macrophage NO production. Moreover, some Echinococcus antigens inhibit in vitro NO production when cells were previously exposed to lipopolisacharide (LPS) stimulation. This inhibitory effect was also seen when Echinococcus multilocularis laminated-layer or cysts wall soluble components from both species were used in the experiment [27]. On the other hand, different effects were observed when macrophages were incubated with antigens from Paragonimus mexicanus and Schistosoma bovis. We have observed that excretory-secretory products from Paragonimus mexicanus adult worms trigger NO production from alveolar macrophages in vitro in a specific and concentration-dependent manner [28]. Our results also demonstrated that the stimulation of NO production by alveolar macrophages was accompanied by an increase in iNOS mRNA detection. In this study, we demonstrated 


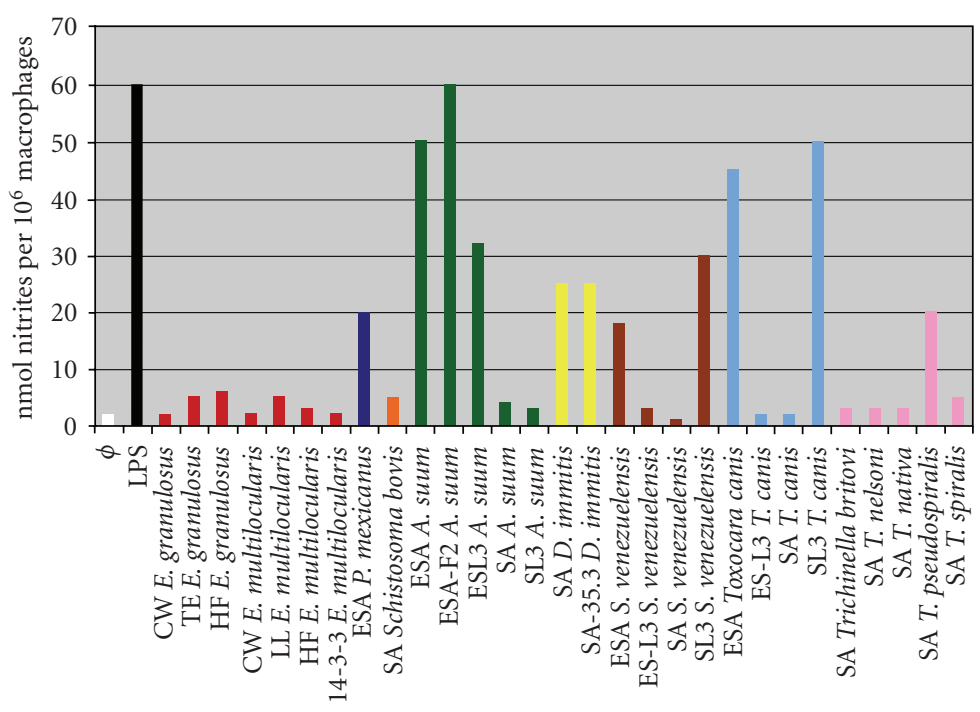

FIGURE 1: Effect of NO production by alveolar macrophages stimulated with different helminth antigens. Nonstimulated ( $\phi)$, LPS-stimulated macrophages (LPS), cyst wall (CW), total extract (TE), hydatid fluid (HF), soluble laminated-layer (LL), E14t recombinant 14-3-3 protein (E14t), excretory-secretory adult (ESA), and somatic adult (SA), excretory-secretory larvae (ESL3), somatic larvae (SL3). Helminths are represented in different colours.

that specific excretory-secretory antigens from Schistosoma bovis adult worms did not induce in vitro NO release from alveolar macrophages. This could be explained because some of these parasites specifically localize in the lungs (e.g., Paragonimus sp.), whereas others migrate through the lungs and definitively settle in other organs (e.g., Schistosoma sp.).

Secondly, there are different effects in species of parasites from the same genus. We investigated the stimulatory/inhibitory role of L1 antigens from four encapsulated (Trichinella spiralis, Trichinella britovi, Trichinella nelsonand Trichinella nativa) and one nonencapsulated (Trichinella pseudospiralis) species of Trichinella on NO production. Our results demonstrated that encapsulated and nonencapsulated Trichinella species differ in their ability to stimulate the NO secretion from the host macrophages [29]. These differences could be related to the complete and incomplete formation of the nurse cells in trichinellosis. Finally, we have studied the effect of different antigens, somatic and excretory/secretory of larval and adult worms of nematodes, on NO production from rat alveolar macrophages. We have observed that somatic antigens from the third stage larvae and excretory/secretory antigens from adult worms in Toxocara canis and Strongyloides venezuelensis stimulated the NO production from alveolar macrophages ([30]; unpublished data); similar data were found with Ascaris suum antigens [31].

We tried to define specific parasite molecules responsible for the stimulation of NO by nematode antigens. Thus, we assayed 10 protein fractions purified from Ascaris suum excretory/secretory adult worm antigens. The fraction-2 included three protein bands with molecular weights of 46 , 44 , and $37 \mathrm{kDa}$. The MALDI-peptide mass fingerprinting analysis showed similarities with two glycolytic enzymes (enolase and phosphoglycerate kinase) and with proteins involved in catalysing the elongation of peptide chains in protein biosynthesis (the elongation factor $\mathrm{Tu}$ ). These proteins are crucial for survival and key components of the protein synthesis machinery [28]. We also analyzed different parasite proteins to define the specific Dirofilaria immitis somatic adult-worm antigens involved in host cell NO secretion. We identified a parasitic specific component (DiID35.3) which does not belong to the endosymbiont Wolbachia and which is intimately related to proteins of the Immunoglobulin Superfamily (ISP) group, triggering NO release from macrophages in a dose-dependent and specific manner [31].

The cellular receptor of antigens, and the intracellular transduction pathways, that trigger $\mathrm{NO}$ production have been precisely identified [32]. Our research group studied the cytoplasmatic signalling pathways involved in the NO production after stimulation with adult excretory/secretory antigen of Toxocara canis [33]. Our results suggested that both phospholipase A2 and phospholipase $\mathrm{C}$ macrophage pathways play an essential role in activating the production of NO triggered by this antigen. This suggests that NO production could be due to an increase of intracellular calcium and activation of the arachidonic acid pathway.

\section{What Is the Biological Effect of NO Production on the Helminths?}

The role of $\mathrm{NO}$ in the defence against the helminths is sustained by various types of studies, such as the utilization of NO donors in different experiments (both in vivo and 
in vitro) and the evaluation of their action on the stages of the biological cycle of the helminths. This strategy has been used to study the effect of this molecule on Echinococcus granulosus, Brugia malayi, and Trichinella spiralis. SNAP donors produced lesions and cell death in Echinococcus granulosus [34]; and similarly, SIN-1 had cytostatic effects in vitro on microfilariae and adult worms of Brugia malayi, being the adults more resistant [35]. Moreover, the use of different donor (DAE/NO) in an experimental model of filariosis inhibits the development of adult worms and alters its motility [36]. On the other hand, it has been observed that different donors of NO increased worm recovery in a trichinellosis experimental model [37, 38]. Moreover, induction of NO synthesis may have a negative effect on the host's immune response in a local environment. This, together with downregulation of mannose receptor expression, could participate in the survival strategy of the Trichinella spiralis in the host [39].

Our research group studied the effects of two NO donors, SIN-1 and SNOG, on Toxocara canis larvae at different concentrations. The results showed that none of the concentrations used from both donors or in combination with oxygen free radicals exerted any cytotoxic effect on Toxocara canis larvae [40]. Therefore, the stimulation of NO production by Toxocara canis larvae antigens does not seem to play any host-defensive role, in contrast to the deleterious effects attributed to this molecule upon other helminths, for example, filarial nematodes. We also utilized NO donors (with short, middle and long half life) to evaluate their effect on larvae and Strongyloides venezuelensis female adult worms where we could demonstrate a dose-dependent cytotoxic effect (unpublished data).

Interaction with oxygen free radicals or immunoglobulins may be considered to evaluate combined effect in host cells. Coculture of Echinococcus multilocularis protoescolices with prestimulated macrophages leads to the destruction of the parasite, being NO the mediator involved [41]. Moreover, peritoneal macrophages stimulated with $\gamma$-IFN cause damage in Echinococcus granulosus, similar to the damage produced by NO donors [34]. A similar effect occurs when Brugia malayi and Onchocerca lienalis microfilariae are cocultured with macrophages activated by $\gamma$-IFN [42]. Finally, other cells like Biomphalaria haemocytes are capable to destroy Schistosoma mansoni sporocysts [43].

NO synthesis inhibitors have been used in vivo to evaluate their effect on parasitic infection. This strategy has mainly been used in experimental models of filariosis by Brugia malayi [36], toxocariosis [40], trichinellosis [44], and strongyloidiasis (unpublished data). The results obtained are divergent, since the use of aminoguanidin diminishes the lesions in toxocariosis, whereas it increases the parasite load in filariosis and strongyloidiasis. Moreover, mice treated with aminoguanidine at the beginning of muscle phase of the infection inhibits the reduction of muscle larvae number [44] and cells of inflammatory infiltrates did not show any specific iNOS reaction [25]. A decrease in eggs in faeces and reduction of larvae in the lung and females in the intestine have been observed in experimental strongyloidiasis. A model of immunosuppression was developed in mice infected with Strongyloides venezuelensis administrated with dexametasone. Our results demonstrated the importance of $\mathrm{NO}$ in the defence against Strongyloides venezuelensis, since the immunosuppressed mice treated with aminoguanidin presented a very significant increase in both, the egg count and the larvae and female recoveries from the lung and intestine. Moreover, the use of antihelminthics (praziquantel, ivermectin and diethylcarbamacine) is associated with an increase in serum concentration of nitrites and nitrates. It has been interpreted that the antiparasitic activity of these drugs depends in part on NO release $[45,46]$.

As conclusion, it is important to highlight that the use of different experimental methods leads to contradictory results. For example, whereas in vitro studies demonstrated the role of NO in the destruction of schistosomules [47], in vivo studies did not support these results [48], probably because schistosomules are protected from the effect of NO by haemoglobin from red blood cells. Moreover, some authors suggested that $\mathrm{NO}$ is involved in the control of lymphatic filariosis $[36,42]$, whereas other question the role of this molecule in the clearance of microfilariae in knock-out mice model. [49].

\section{What Pathological Consequences Are Derived from the Production of Nitric Oxide in the Host?}

NO production by the host cells in response to helminth infections can cause adverse effects. Direct lesions or tissue functional alterations, immunosuppression, and carcinogenesis are the principal consequences. Trichinellosis [5052], schistosomiasis and dirofilariosis [3] are the main examples of structural and functional direct lesions. It has been demonstrated that $\mathrm{NO}$ released in response to some helminths leads to immunosuppression, with low proliferative response of splenocytes or apoptosis induction of CD4 lymphocytes. Infections by Echinococcus multilocularis [22, 53] and filariosis [54] are the best characterised examples. Finally, the release of NO in response to the infection by $S$. haematobium seems to play an important role in the bladder cancer, through modifications of the p53 protein [55].

Our research group studied the influence of the inhibition of the NO production in a toxocariosis experimental model. The results clearly suggested that in vivo inhibition of the NO synthesis by iNOS decreases the deleterious effects of the parasite upon the host, especially the lung vascular alterations. We could show that in vivo NO production induced by infection with Toxocara canis results in direct damage to the host. This induction constitutes an evasion/adaptation mechanism of the parasite [40]. Moreover, Toxocara canis excretory/secretory adult antigen also stimulated alveolar macrophages to produce prostaglandin $\mathrm{E}_{2}\left(\mathrm{PGE}_{2}\right)$. The addition of $\mathrm{L}$-canavanine decreased the release of $\mathrm{PGE}_{2}$, which suggests that NO mediates the production of this molecule [30]. These results indicate that Toxocara canis can stimulate the release of vasodilatory mediators by host macrophages. 
It is also important to indicate that in some helminthic diseases both excess and lack of $\mathrm{NO}$ can have deleterious effects for the host [56-58]. Excessive production of NO in schistosomiasis unleashes an acute response with direct hepatotoxicity and formation of granulomas with scarce fibrosis. However, a low NO production of this mediator is associated to chronic evolution of schistosomiasis with the development of intense fibrosis and granulomas of great size.

\section{Conclusions and Future Perspectives}

Nitrites or NOS has been detected in helminths at different stages of their biological cycle and in distinct anatomical structures. Stimulation of cells involved in the defence of the respiratory system (alveolar macrophages) with antigens from different biological stages of helminths has demonstrated that (i) antigens of cestodes inhibit the NO production, (ii) antigens of trematodes have different effects on its production, and (iii) antigens of nematodes stimulate the production of this mediator. It has been demonstrated that somatic antigens of larvae and excretory/secretory of adult worms induce the NO production in nematodes such as Toxocara or Strongyloides. Moreover, there are differences between the cystic and noncystic species of Trichinella in their capacity to stimulate the production of nitric oxide. These differences could be related to the complete and incomplete formation of the nurse cells in trichinellosis.

In an attempt to advance in the study of the molecules responsible for the stimulation of $\mathrm{NO}$ production, two glucolytic enzymes (enolase and phosphoglycerate kinase) and a protein that catalyses the elongation of peptide chains named Tu elongation factor have been identified in A. suum. A protein of $D$. immitis has also been recognised which is related to the Immunoglobulin Superfamily Protein (ISP).

The information obtained regarding the biological effects that NO production unleashes on the parasite or on the host is contradictory. NO is not harmful on Toxocara canis, whereas it kills larvae and adults of Strongyloides venezuelensis. In addition, NO plays an important role in the defence against strongyloidosis; its absence might even play a role in hyperinfection syndrome caused by this parasite. Furthermore, NO production constitutes an evasion mechanism in toxocariosis.

Finally and given that (i) VEGF (vascular endothelium growth factor) and FGF2 (fibroblastic growth factor) are important molecules in angiogenesis, performing other essential functions in inflammation (e.g., chemotaxis), (ii) alveolar macrophages, and specifically human alveolar macrophages, express VEGF, (iii) NO and VEGF are molecules with an intimate functional relationship, (iv) angiogenesis plays an important role in some helminthic diseases (schistosomiasis, onchocercosis, trichinellosis), and (v) the role of the helminths in the production of these factors is not well known, the study of the production of angiogenic factors in the respiratory helminthic diseases and the analysis of their biological effects would be of great interest to the knowledge of the pathogenic mechanisms of these infections.

\section{Acknowledgments}

AGL-2005-02168, AGL2008-03187/GAN, FISPI061355, and Junta de Castilla y León SA116A08 Grants supported this work. Doctoral and postdoctoral fellowships have been supported by CNPq-Ministerio de Educación de Brasil, Fundación Carolina, and Universidad de Salamanca-Banco Santander. This work is composed of results already published (Parasite Immunology 2002 a, b, c; 2005; Nitric Oxide 2004, 2005, Experimental Parasitology 2005, Veterinary Parasitolology 2007) and unpublished data. The authors express their gratitude to all the people who have participated in these works, since without them it would not have been possible to develop this research, specially E. Espinoza Ph.D., M.A. Andrade Ph.D. and A.L. Ruano M.D., Ph.D. The authors would also like to thank Mr. G.H. Jenkins for his help with the English version of the manuscript. They gratefully acknowledge the contributions of Dr. J. Pérez Losada and Dr. A. Castellanos in the reviewing of the manuscript.

\section{References}

[1] A. B. Kohn, L. L. Moroz, J. M. Lea, and R. M. Greenberg, "Distribution of nitric oxide synthase immunoreactivity in the nervous system and peripheral tissues of Schistosoma mansoni," Parasitology, vol. 122, no. 1, pp. 87-92, 2001.

[2] X.-C. Long, M. Bahgat, K. Chlichlia, A. Ruppel, and Y.-L. Li, "Detection of inducible nitric oxide synthase in Schistosoma japonicum and S. mansoni," Journal of Helminthology, vol. 78, no. 1, pp. 47-50, 2004.

[3] K. Kitoh, A. Oka, H. Kitagawa, T. Unno, S. Komori, and Y. Sasaki, "Relaxing and contracting activities of heartworm extract on isolated canine abdominal aorta," Journal of Parasitology, vol. 87, no. 3, pp. 522-526, 2001.

[4] Z. A. Bascal, A. Montgomery, L. Holden-Dye, R. G. Williams, and R. J. Walker, "Histochemical mapping of NADPH diaphorase in the nervous system of the parasitic nematode Ascaris suum," Parasitology, vol. 110, no. 5, pp. 625-637, 1995.

[5] J. W. Bowman, C. A. Winterrowd, A. R. Friedman, et al., "Nitric oxide mediates the inhibitory effects of SDPNFLRFamide, a nematode FMRFamide-related neuropeptide, in Ascaris suum," Journal of Neurophysiology, vol. 74, no. 5, pp. 1880-1888, 1995.

[6] Z. A. Bascal, J. M. Cunningham, L. Holden-Dye, M. O’Shea, and R. J. Walker, "Characterization of a putative nitric oxide synthase in the neuromuscular system of the parasitic nematode, Ascaris suum," Parasitology, vol. 122, no. 2, pp. 219231, 2001.

[7] C.-K. Fan, Y.-H. Lin, C.-C. Hung, S.-F. Chang, and K.-E. $\mathrm{Su}$, "Enhanced inducible nitric oxide synthase expression and nitrotyrosine accumulation in experimental granulomatous hepatitis caused by Toxocara canis in mice," Parasite Immunology, vol. 26, no. 6-7, pp. 273-281, 2004.

[8] K. M. Pfarr and J. A. Fuhrman, "Brugia malayi: localization of nitric oxide synthase in a lymphatic filariid," Experimental Parasitology, vol. 94, no. 2, pp. 92-98, 2000.

[9] K. M. Pfarr, S. Qazi, and J. A. Fuhrman, "Nitric oxide synthase in filariae: demonstration of nitric oxide production by embryos in Brugia malayi and Acanthocheilonema viteae," Experimental Parasitology, vol. 97, no. 4, pp. 205-214, 2001.

[10] M. Masetti, T. Locci, A. Cecchettini, et al., "Nitric oxide synthase immunoreactivity in the nematode Trichinella britovi. 
Evidence for nitric oxide production by the parasite," International Journal for Parasitology, vol. 34, no. 6, pp. 715-721, 2004.

[11] R. Amanvermez and C. Celik, "Effectiveness of free radicals in hydatid cysts," Journal of the Egyptian Society of Parasitology, vol. 32, no. 1, pp. 259-269, 2002.

[12] A. Martínez, "Nitric oxide synthase in invertebrates," Histochemical Journal, vol. 27, no. 10, pp. 770-776, 1995.

[13] J. W. Bowman, A. R. Friedman, D. P. Thompson, A. G. Maule, S. J. Alexander-Bowman, and T. G. Geary, "Structure-activity relationships of an inhibitory nematode FMRFamide-related peptide, SDPNFLRFamide (PF1), on Ascaris suum muscle," International Journal for Parasitology, vol. 32, no. 14, pp. 17651771, 2002.

[14] B. Kuzin, I. Roberts, N. Peunova, and G. Enikolopov, "Nitric oxide regulates cell proliferation during Drosophila development," Cell, vol. 87, no. 4, pp. 639-649, 1996.

[15] S. M. Gibbs and J. W. Truman, "Nitric oxide and cyclic GMP regulate retinal patterning in the optic lobe of Drosophila," Neuron, vol. 20, no. 1, pp. 83-93, 1998.

[16] S. J. Froggett and E. M. Leise, "Metamorphosis in the marine snail Ilyanassa obsoleta, yes or NO?" Biological Bulletin, vol. 196, no. 1, pp. 57-62, 1999.

[17] D. M. Minning, A. J. Gow, J. Bonavetura, et al., "Ascaris haemoglobin is a nitric oxide-activated 'deoxygenase', Nature, vol. 401, no. 6752, pp. 497-502, 1999.

[18] J. Barrett and P. M. Brophy, "Ascaris haemoglobin: new tricks for an old protein," Parasitology Today, vol. 16, no. 3, pp. 90 91, 2000.

[19] C. Touil-Boukoffa, B. Bauvois, J. Sancéau, B. Hamrioui, and J. Wietzerbin, "Production of nitric oxide (NO) in human hydatidosis: relationship between nitrite production and interferon- $\gamma$ levels," Biochimie, vol. 80, no. 8-9, pp. 739$744,1998$.

[20] S. Abo-Shousha, S. S. Khalil, and E. A. Rashwan, "Oxygen free radical and nitric oxide production in single or combined human schistosomiasis and fascioliasis," Journal of the Egyptian Society of Parasitology, vol. 29, no. 1, pp. 149-156, 1999.

[21] S. Ait Aissa, M. Amri, R. Bouteldja, J. Wietzerbin, and C. Touil-Boukoffa, "Alterations in interferon-gamma and nitric oxide levels in human echinococcosis," Cellular and Molecular Biology, vol. 52, no. 1, pp. 65-70, 2006.

[22] W. J. Dai and B. Gottstein, "Nitric oxide-mediated immunosuppression following murine Echinococcus multilocularis infection," Immunology, vol. 97, no. 1, pp. 107-116, 1999.

[23] M. Hirata, K. Hirata, M. Kage, M. Zhang, T. Hara, and T. Fukuma, "Effect of nitric oxide synthase inhibition on Schistosoma japonicum egg-induced granuloma formation in the mouse liver," Parasite Immunology, vol. 23, no. 6, pp. 281$289,2001$.

[24] C. M. Hogaboam, S. M. Collins, and M. G. Blennerhassett, "Effects of oral L-NAME during Trichinella spiralis infection in rats," American Journal of Physiology, vol. 271, no. 2, pp. G338G346, 1996.

[25] J. Zeromski, K. Boczoń, E. Wandurska-Nowak, and I. MozerLisewska, "Effect of aminoguanidine and albendazole on inducible nitric oxide synthase (iNOS) activity in T. spiralisinfected mice muscles," Folia Histochemica et Cytobiologica, vol. 43, no. 3, pp. 157-159, 2005.

[26] Y. Chen and D. L. Boros, "Polarization of the immune response to the single immunodominant epitope of p38, a major Schistosoma mansoni egg antigen, generates Th1- or Th2-type cytokines and granulomas," Infection and Immunity, vol. 67, no. 9, pp. 4570-4577, 1999.
[27] M. A. Andrade, M. Siles-Lucas, E. Espinoza, J.-L. PérezArellano, B. Gottstein, and A. Muro, "Echinococcus multilocularis laminated-layer components and the E14t 14-3-3 recombinant protein decrease $\mathrm{NO}$ production by activated rat macrophages in vitro," Nitric Oxide, vol. 10, no. 3, pp. 150$155,2004$.

[28] M. A. Andrade, M. Siles-Lucas, J. López-Abán, J.-L. PérezArellano, I. D. Vélez, and A. Muro, "Lung-migrating digenean parasites: in vitro influence on nitric oxide production from normal rat pulmonary macrophages," Experimental Parasitology, vol. 109, no. 3, pp. 171-175, 2005.

[29] M. A. Andrade, M. Siles-Lucas, J. López-Abán, et al., "Trichinella: differing effects of antigens from encapsulated and non-encapsulated species on in vitro nitric oxide production," Veterinary Parasitology, vol. 143, no. 1, pp. 86-90, 2007.

[30] E. Espinoza, A. Muro, M.-M. Sánchez Martín, P. Casanueva, and J.-L. Pérez-Arellano, "Toxocara canis antigens stimulate the production of nitric oxide and prostaglandin $\mathrm{E}_{2}$ by rat alveolar macrophages," Parasite Immunology, vol. 24, no. 6, pp. 311-319, 2002.

[31] M. A. Andrade, M. Siles-Lucas, J. López-Abán, C. Carranza, J.-L. Pérez-Arellano, and A. Muro, "Antigens from Ascaris suum trigger in vitro macrophage NO production," Parasite Immunology, vol. 27, no. 6, pp. 235-242, 2005.

[32] H. Tezuka, S. Imai, S. Tsukidate, and K. Fujita, "A Dirofilaria immitis polyprotein up-regulates nitric oxide production," Infection and Immunity, vol. 70, no. 9, pp. 5283-5286, 2002.

[33] E. Espinoza, J.-L. Pérez-Arellano, B. Vicente, and A. Muro, "Cytoplasmic signalling pathways in alveolar macrophages involved in the production of nitric oxide after stimulation with excretory/secretory antigens of Toxocara canis," Parasite Immunology, vol. 24, no. 11-12, pp. 535-544, 2002.

[34] N. J. R. Steers, M. T. Rogan, and S. Heath, "In-vitro susceptibility of hydatid cysts of Echinococcus granulosus to nitric oxide and the effect of the laminated layer on nitric oxide production," Parasite Immunology, vol. 23, no. 8, pp. 411-417, 2001.

[35] G. R. Thomas, M. McCrossan, and M. E. Selkirk, "Cytostatic and cytotoxic effects of activated macrophages and nitric oxide donors on Brugia malayi," Infection and Immunity, vol. 65, no. 7, pp. 2732-2739, 1997.

[36] T. V. Rajan, P. Porte, J. A. Yates, L. Keefer, and L. D. Shultz, "Role of nitric oxide in host defense against an extracellular, metazoan parasite, Brugia malayi," Infection and Immunity, vol. 64, no. 8, pp. 3351-3353, 1996.

[37] E. Hadaś, M. Derda, and E. Wandurska-Nowak, "Effect of exogenous nitric oxide in experimental trichinellosis," Parasitology Research, vol. 88, no. 1, pp. 86-88, 2002.

[38] E. Wandurska-Nowak, E. Hadaś, M. Derda, and W. Wojt, "Effect of nitric oxide releasing drugs on the intensity of infection during experimental trichinellosis in mice," Parasitology Research, vol. 90, no. 2, pp. 164-165, 2003.

[39] A. Gruden-Movsesijan and L. S. Milosavljevic, "The involvement of the macrophage mannose receptor in the innate immune response to infection with parasite Trichinella spiralis," Veterinary Immunology and Immunopathology, vol. 109, no. 1-2, pp. 57-67, 2006.

[40] E. Espinoza, J.-L. Pérez-Arellano, C. Carranza, F. Collía, and A. Muro, "In vivo inhibition of inducible nitric oxide synthase decreases lung injury induced by Toxocara canis in experimentally infected rats," Parasite Immunology, vol. 24, no. 11-12, pp. 511-520, 2002.

[41] T. Kanazawa, H. Asahi, H. Hata, K. Mochida, N. Kagei, and M. J. Stadecker, "Arginine-dependent generation of reactive 
nitrogen intermediates is instrumental in the in vitro killing of protoscoleces of Echinococcus multilocularis by activated macrophages," Parasite Immunology, vol. 15, no. 11, pp. 619623, 1993.

[42] M. J. Taylor, H. F. Cross, A. A. Mohammed, A. J. Trees, and A. E. Bianco, "Susceptibility of Brugia malayi and Onchocerca lienalis microfilariae to nitric oxide and hydrogen peroxide in cell-free culture and from IFN $\gamma$-activated macrophages," Parasitology, vol. 112, no. 3, pp. 315-322, 1996.

[43] U. K. Hahn, R. C. Bender, and C. J. Bayne, "Involvement of nitric oxide in killing of Schistosoma mansoni sporocysts by hemocytes from resistant Biomphalaria glabrata," Journal of Parasitology, vol. 87, no. 4, pp. 778-785, 2001.

[44] M. Kołodziej-Sobocińska, E. Dziemian, and B. MachnickaRowińska, "Inhibition of nitric oxide production by aminoguanidine influences the number of Trichinella spiralis parasites in infected "low responders" (C57BL/6) and "high responders" (BALB/c) mice," Parasitology Research, vol. 99, no. 2, pp. 194-196, 2006.

[45] E. M. Ammar, S. A. Said, and L. A. Eissa, "Mediation of the schistosomicidal effect of praziquantel through nitric oxide," Arzneimittel-Forschung, vol. 52, no. 11, pp. 844-848, 2002.

[46] S. Winkler, I. El Menyawi, K. F. Linnau, and W. Graninger, "Short report: total serum levels of the nitric oxide derivatives nitrite/nitrate during microfilarial clearance in human filarial disease," American Journal of Tropical Medicine and Hygiene, vol. 59, no. 4, pp. 523-525, 1998.

[47] S. L. James and J. Glaven, "Macrophage cytotoxicity against schistosomula of Schistosoma mansoni involves argininedependent production of reactive nitrogen intermediates," Journal of Immunology, vol. 143, no. 12, pp. 4208-4212, 1989.

[48] P. S. Coulson, L. E. Smythies, C. Betts, et al., "Nitric oxide produced in the lungs of mice immunized with the radiationattenuated schistosome vaccine is not the major agent causing challenge parasite elimination," Immunology, vol. 93, no. 1, pp. 55-63, 1998.

[49] C. A. Gray and R. A. Lawrence, "Interferon- $\gamma$ and nitric oxide production are not required for the immune-mediated clearance of Brugia malayi microfilariae in mice," Parasite Immunology, vol. 24, no. 6, pp. 329-336, 2002.

[50] C. E. Lawrence, J. C. M. Paterson, X.-Q. Wei, F. Y. Liew, P. Garside, and M. W. Kennedy, "Nitric oxide mediates intestinal pathology but not immune expulsion during Trichinella spiralis infection in mice," Journal of Immunology, vol. 164, no. 8, pp. 4229-4234, 2000.

[51] P. Garside, M. W. Kennedy, D. Wakelin, and C. E. Lawrence, "Immunopathology of intestinal helminth infection," Parasite Immunology, vol. 22, no. 12, pp. 605-612, 2000.

[52] D. Torrents, N. Prats, and P. Vergara, "Inducible nitric oxide synthase inhibitors ameliorate hypermotility observed after $T$. spiralis infection in the rat," Digestive Diseases and Sciences, vol. 48, no. 6, pp. 1035-1049, 2003.

[53] W. J. Dai, A. Waldvogel, T. Jungi, M. Stettler, and B. Gottstein, "Inducible nitric oxide synthase deficiency in mice increases resistance to chronic infection with Echinococcus multilocularis," Immunology, vol. 108, no. 2, pp. 238-244, 2003.

[54] J. S. Jenson, R. O’Connor, J. Osborne, and E. Devaney, "Infection with Brugia microfilariae induces apoptosis of $\mathrm{CD}^{+} \mathrm{T}$ lymphocytes: a mechanism of immune unresponsiveness in filariasis," European Journal of Immunology, vol. 32, no. 3, pp. 858-867, 2002.

[55] W. Warren, P. J. Biggs, M. El-Baz, M. A. Ghoneim, M. R. Stratton, and S. Venitt, "Mutations in the p53 gene in schistosomal bladder cancer: a study of 92 tumours from Egyptian patients and a comparison between mutational spectra from schistosomal and non-schistosomal urothelial tumours," Carcinogenesis, vol. 16, no. 5, pp. 1181-1189, 1995.

[56] K. F. Hoffmann, A. W. Cheever, and T. A. Wynn, "IL10 and the dangers of immune polarization: excessive type 1 and type 2 cytokine responses induce distinct forms of lethal immunopathology in murine schistosomiasis," Journal of Immunology, vol. 164, no. 12, pp. 6406-6416, 2000.

[57] A. C. La Flamme, E. A. Patton, B. Bauman, and E. J. Pearce, "IL-4 plays ,a crucial role in regulating oxidative damage in the liver during schistosomiasis," Journal of Immunology, vol. 166, no. 3, pp. 1903-1911, 2001.

[58] E. A. Patton, A. C. La Flamme, J. A. Pedras-Vasoncelos, and E. J. Pearce, "Central role for interleukin-4 in regulating nitric oxide-mediated inhibition of T-cell proliferation and gamma interferon production in schistosomiasis," Infection and Immunity, vol. 70, no. 1, pp. 177-184, 2002. 

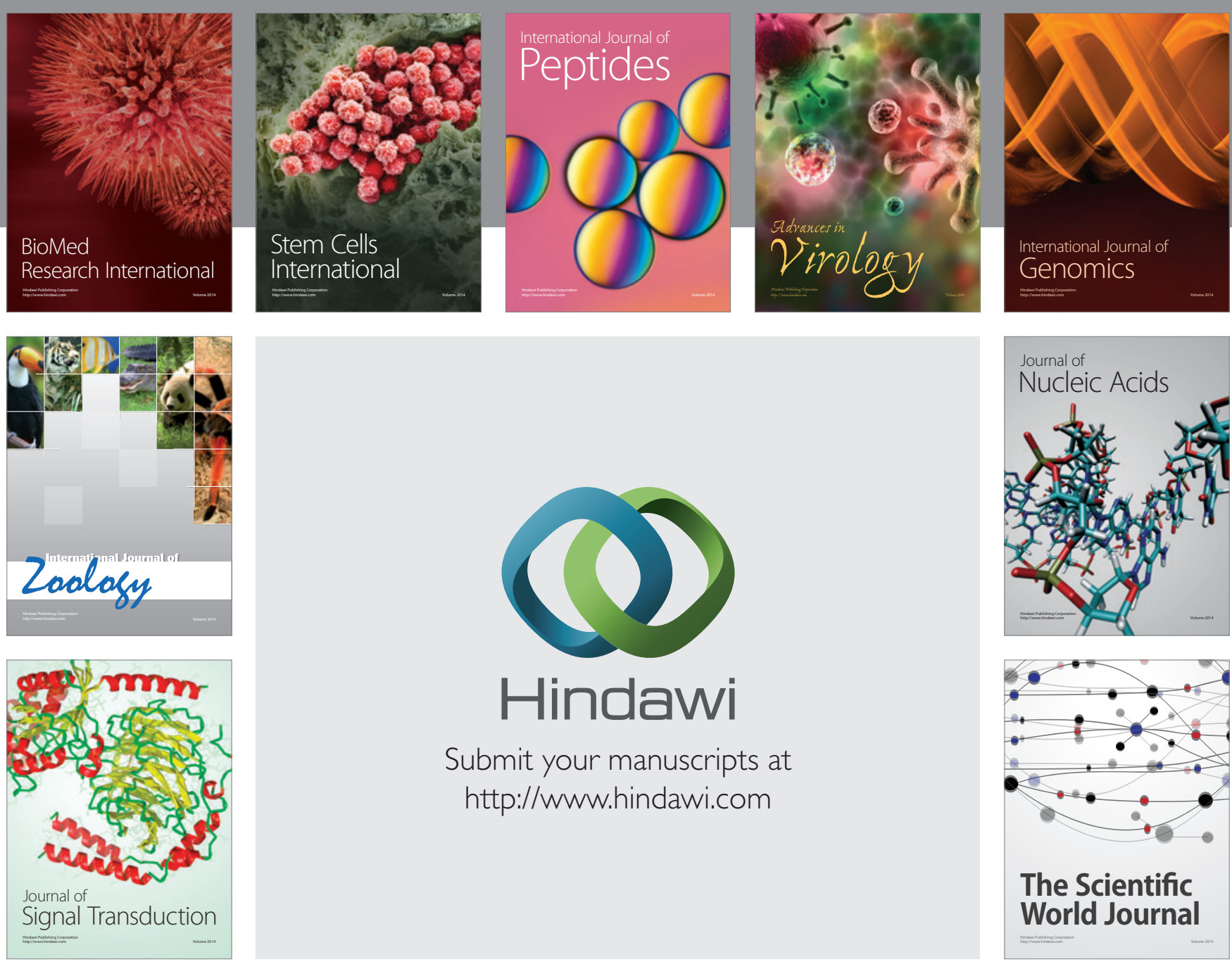

Submit your manuscripts at

http://www.hindawi.com
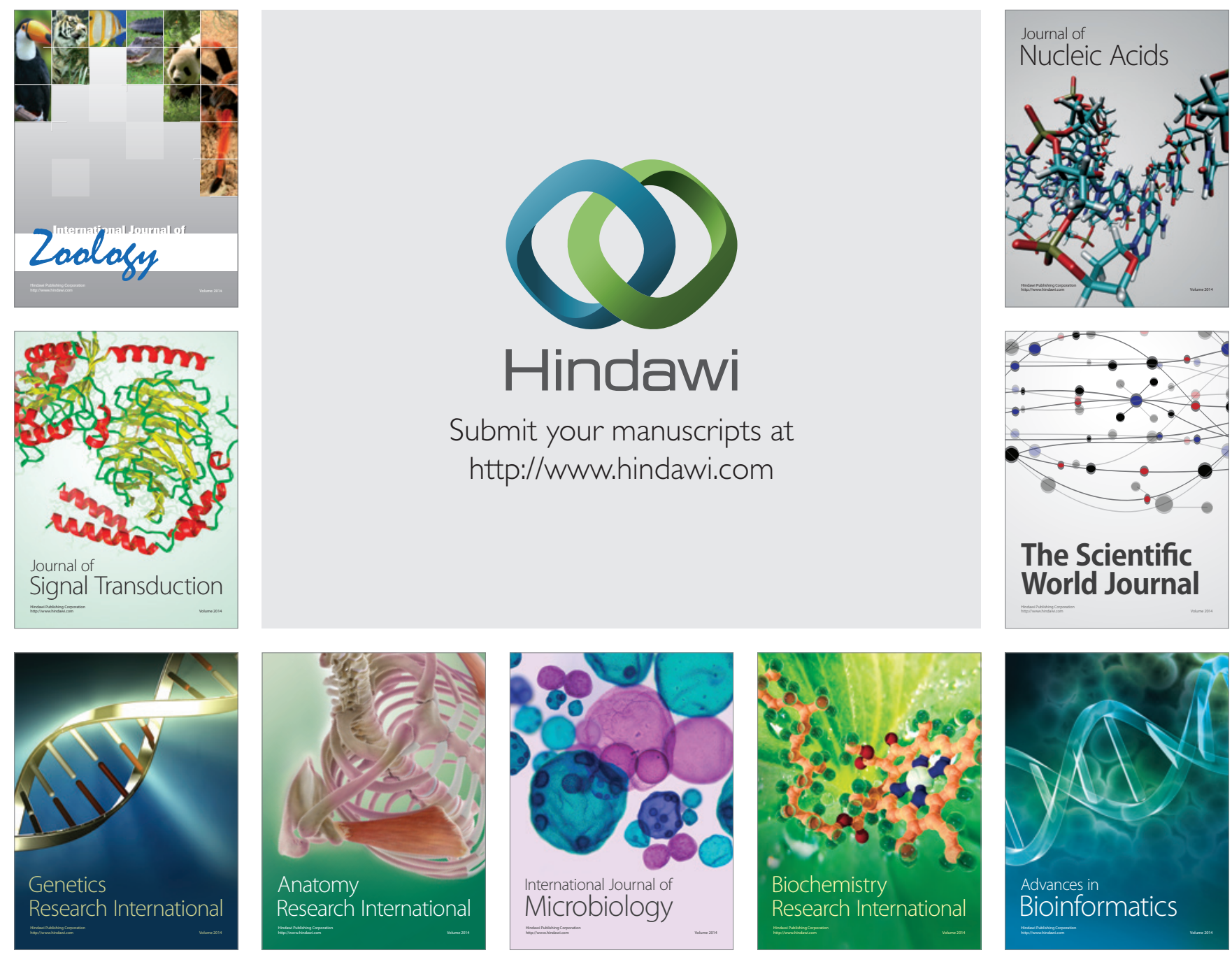

The Scientific World Journal
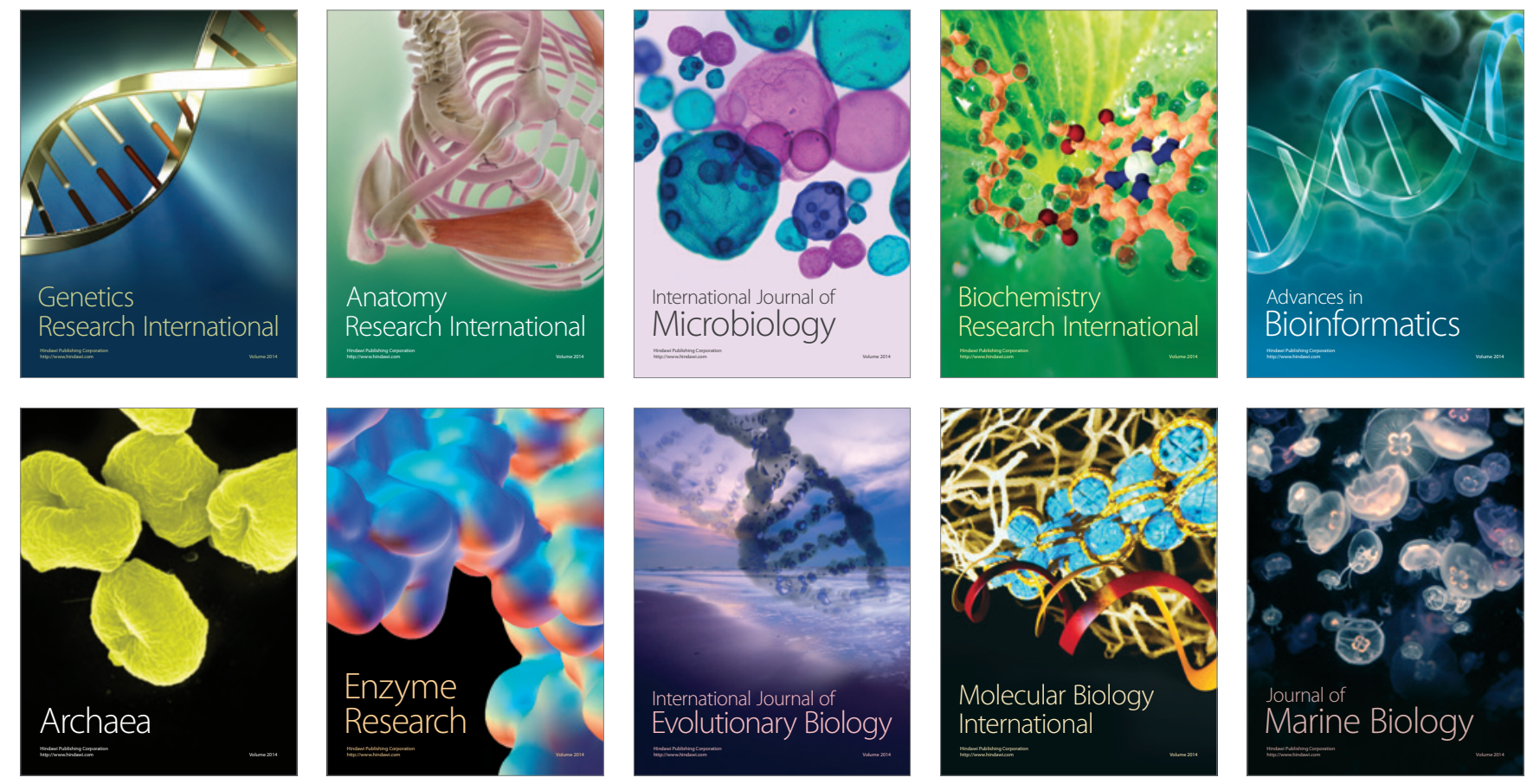